\title{
Strategies for reducing the use of greenhouse gases from particle detectors operation at the CERN LHC experiments
}

\author{
R. Guida, ${ }^{a, *}$ M. Corbetta, ${ }^{a, b}$ B. Mandelli ${ }^{a}$ and G. Rigoletti ${ }^{a, b}$ \\ a CERN, \\ Espl. des Particules 1, Meyrin, Switzerland \\ ${ }^{b}$ University Claude Bernard Lyon I, \\ Lyon, France \\ E-mail: roberto.guida@cern.ch
}

A wide range of gas mixtures is used for the operation of different gaseous detectors for particle physics research. Among them are greenhouse gases like $\mathrm{C}_{2} \mathrm{H}_{2} \mathrm{~F}_{4}(\mathrm{R} 134 \mathrm{a}), \mathrm{CF}_{4}(\mathrm{R} 14), \mathrm{C}_{4} \mathrm{~F}_{10}$ (R610) and $\mathrm{SF}_{6}$, which are used because they allow to achieve specific detector performance that are necessary for data taking at the LHC experiments (i.e. stability, long term performance, time resolution, rate capability, etc.). Such gases are currently subject to a phase down policy that started to affect the market with price increase and, in the long term, may cause a decrease in their availability. Four different strategies have been identified to optimize the gas usage. As immediate actions, during the LHC Long Shutdown 2 the gas systems will be upgraded to cope with new detector requirements and, in parallel, extensive campaigns for fixing leaks at detector level will be performed. The development of gas recuperation plants is going to be the next step. They aim in extracting greenhouse gases from the exhaust of gas recirculation systems allowing further re-use. Several plants of this type are already in use at the CERN LHC experiments. Recent developments are concerning systems for $\mathrm{CF}_{4}$ and $\mathrm{R} 134 \mathrm{a}$ recuperation. Recently two systems for $\mathrm{CF}_{4}$ were developed allowing to reduce the consumption by about 50\%. The R134a recuperation plant is still in a $R \& D$ phase; however, encouraging results have been obtained. For future long-term detector operation, $R \& D$ studies are ongoing for finding green alternatives to the currently used gases. The last strategy consists in using industrially developed plants for the disposal of greenhouse gases by decomposition in harmless compounds. This solution avoids the emission in the atmosphere but it is not optimizing the gas usage. Moreover, problems like gas availability and price are not addressed and they might become the challenge in the years to come due to the greenhouse phase down policy.

40th International Conference on High Energy physics - ICHEP2020

July 28 - August 6, 2020

Prague, Czech Republic (virtual meeting)

\footnotetext{
${ }^{*}$ Speaker
} 


\section{Introduction}

A wide range of gas mixtures is used for the operation of different gaseous detectors for particle physics research. Some of these gases are recognized as greenhouse gases (GHGs). Unfortunately, at the moment particle detectors are forced to use them because they allow to achieve specific detector performance that are absolutely needed for data taking at the LHC experiments (i.e. stability, long term performance, time resolution, rate capability, etc.). GHGs are classified according to their Global Warming potential (GWP). Gases like $\mathrm{C}_{2} \mathrm{H}_{2} \mathrm{~F}_{4}$ (R134a), $\mathrm{CF}_{4}(\mathrm{R} 14), \mathrm{C}_{4} \mathrm{~F}_{10}$ (R610) and $\mathrm{SF}_{6}$, used for operation of particle detectors are characterized by very high global warming potential (from 1430 to 22800). In addition, it is fundamental to consider that in the European Union, GHGs with high GWP are already subject to a phase down policy that started to affect the market with price increase and, at long term, it might cause a decrease in the availability. The EU regulation on fluorinated gases is aiming in limiting the total amount of F-gases that can be sold in EU with a reduction to one-fifth of the quantity sold in 2014 by 2030. Moreover, it bans the use of F-gases where less harmful alternatives are available, and it requires adequate checks, maintenance and recovery of the gases to avoid emissions from existing equipment [1].

Reducing the use of GHGs is a worldwide objective to which CERN wants to contribute. In this context and with the idea of preparing the very long-term operation, the CERN EP-DT Gas Team, the CERN Environmental Protection Steering board (CEPS) and the LHC experiments have elaborated a strategy based on several action lines.

\section{2. $R \& D$ strategies}

At the CERN Large Hadron Collider (LHC) experiments 30 gas systems (corresponding to about 300 modules) are delivering the proper gas mixture to the corresponding detectors [2]. Particle detectors at the LHC experiments are very often characterized by large detector volume (up to several hundred cubic meters) and by the need of using very specific gases often expensive and/or GHGs. For these reasons, since the early phase, the gas consumption optimization was a design criteria for reducing operational costs. However, gas recirculation systems are more complex than open mode gas systems (in the latter the gas mixture is vented to the atmosphere after being used into the detectors). In presence of gas recirculation, a constant monitoring of mixture composition and concentration of impurities is mandatory. A dedicated module for gas mixture purification is needed to avoid any impurity accumulation $[4,5]$.

Figure 1 shows the GHG emissions from the LHC experiments during Run1 and Run2. RPC detectors are the major contributors and, therefore, R134a (the main component of the RPC detector mixture) represents the largest part of the GHGs emitted. Recently CERN has published the Environment Report for the years 2017-2018 where also the GHG emission is addressed [6].

Optimization of current technologies Two relatively small systems using GHGs (originally designed in open mode) have been upgraded to gas recirculation during the LHC Long Shutdown 1 (LS1): the RPC muon trigger of ALICE (ALICE-MTR) and the LHCb-GEM muon system (LHCb-GEM).

Thanks to the ALICE-MTR gas system upgrade, the R134a and $\mathrm{SF}_{6}$ consumption from this system has been reduced by about $75 \%$ and the budget invested for the upgrade has been largely 


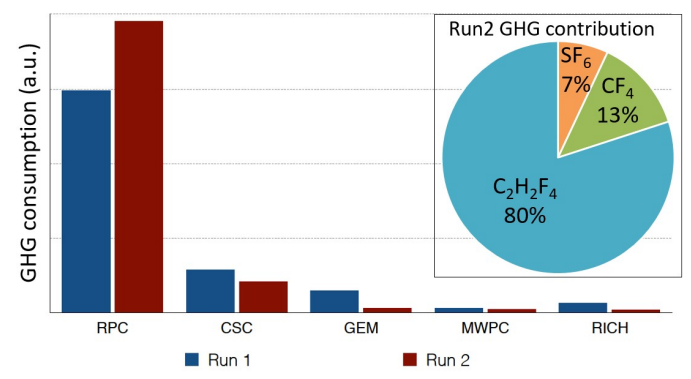

Figure 1: GHG emissions from detector systems at the LHC experiments. The major contribution is due to the RPC detectors (ATLAS and CMS systems) and, therefore, R134a represents $80 \%$ of the GHG emissions in terms of $\mathrm{CO}_{2}$ eq. From Run1 to Run2 the reduction for the GEM system (upgrade to mixture recirculation of LHCb-GEM) is clearly visible while the increase for the RPC systems is due to an increase of the leak rate at detector level.

paid back by the cost saving during operation. However, due to a different working regime with respect to the ATLAS and CMS RPC systems, in the ALICE-MTR detector the pulse charges are higher and, therefore, the formation of impurities due to the fragmentation of the main mixture components was observed. Their concentration was carefully monitored during the run periods and effects on detector performance have been excluded [3].

Concerning LHCb-GEM, since GEM detector had never been operated with gas recirculation before, a dedicated R\&D started in 2013. Given the positive test results, the new LHCb-GEM gas recirculation system was built and became operational in 2016. Since then, a 90\% reduction of GHG has been obtained. Also in this case, the cost for the upgrade has been largely paid back with the cost saving during Run2 operation. In addition, GEM detector performance become more stable thanks to a less frequent replacement of $\mathrm{CF}_{4}$ supply cylinders that in the previous years was causing detector gain fluctuations up to $50 \%$.

Nowadays all detectors employing GHGs are operated with gas recirculation systems. The next step in the optimization aims in improving flow and pressure regulation beyond original requirements to cope with new detectors needs (i.e. enhanced stability, increase of recirculation fraction and flows). For example, during Run 2 the mixture recirculation rate of the RPC detector systems was limited to $85-90 \%$ due to the presence of leaks at the detector level. Despite the difficult challenge, LS2 gives a unique chance to repair as many leaks as possible. However, even if leaks will be repaired, past tests have validated RPC operation at LHC only up to $90 \%$ mixture recirculation. Further tests will be needed to validate stable long-term operation at HL-LHC conditions at the highest possible recirculation rate. Upgrading the gas systems to compensate for the observed detector fragility requires redesigning the gas distribution systems. Several tests have been performed on the gas distribution modules. A new mechanism ensuring a slow opening of the input was introduced in 2016: it reduced by about $70 \%$ the pressure spike during transients. However, at startup the detector is often over pressurized due to the loss of hydrostatic pressure caused by detector leaks. To mitigate this effect the installation of new regulation valves and pressure sensors is ongoing. The latter are mounted on reference volumes simulating RPC detectors at the highest point of each distribution sector. Moreover, new distribution modules will be equipped with regulation valves at the input allowing an opening as smooth as the detector might need. After 
a modification of the mixture return circuit in two distribution modules and an optimization of the regulation loop, an average $70 \%$ improvement of pressure regulation stability was achieved. However, it was not possible to correlate these results with an improvement in the leak development rate. The gas system startup procedure has been modified to minimize pressure fluctuations during re-filling after shutdown periods or any change of gas used for the operation.

Gas recuperation systems The second research line is based on the development of systems able to collect the used gas mixture and to extract GHGs for their re-use. GHG recuperation systems permit to avoid venting to atmosphere gas from the system to allow for the injection of fresh gas. This injection is needed to maintain under control (for a safe and long term detector operation) the concentration of impurities that cannot be filtered. Gas recuperation plants introduce a further additional level of complexity with respect to gas recirculation systems. Moreover, a dedicated $\mathrm{R} \& \mathrm{D}$ is needed for designing a recuperation plant for a specific gas as well as for the definition and tuning of mixture monitoring tools.

Recuperation systems for different gases were developed in the past and are nowadays operational for several detectors: CMS-CSC $\left(\mathrm{CF}_{4}\right)$, ATLAS-TGC $\left(\mathrm{nC}_{5} \mathrm{H}_{12}\right)$, LHCb-RICH1 $\left(\mathrm{C}_{4} \mathrm{~F}_{10}\right)$ and $\mathrm{LHCb}-\mathrm{RICH} 2\left(\mathrm{CF}_{4}\right)$. For most recuperation plants, the mixture returning from the detector is cooled down until the liquefaction point of gas to be extracted is reached. Then the liquid is recuperated and stored. The recuperation efficiency is usually $95 \%$ or higher.

A different method was developed for the $\mathrm{CF}_{4}$ recuperation [7] for the CMS-CSC detector mixture. Due to the very low $\mathrm{CF}_{4}$ boiling point $\left(-128^{\circ} \mathrm{C}\right.$ ) cryogenic liquid (like liquid $\mathrm{N}_{2}$ ) would have been needed. On the contrary, the developed plant makes use of gas separation membranes and selective adsorption during pressure swing cycles in different molecular sieves. The $\mathrm{CF}_{4}$ recuperation plant started operation in 2012. Recently a $44 \%$ reduction of $\mathrm{CF}_{4}$ consumption has been achieved. Studies are ongoing to further improve the performance. A similar systems has been developed and tested for the recuperation of $\mathrm{CF}_{4}$ from the $\mathrm{LHCb}-\mathrm{RICH} 2$ detector. During the first validation test for the design, a $65 \%$ recuperation efficiency has been achieved with a prototype and now the final system is under construction.

$R \& D$ studies are ongoing for the design of a $R 134$ a recuperation plant (R134a represents the large part of GHG emitted). Figure 2 shows a picture and a schematic view of the prototype built in 2018 and connected to the ATLAS-RPC detector and gas systems. The first possibility would be to separate the mixture profiting of the difference in boiling points between $\mathrm{R} 134 \mathrm{a}\left(-26.3{ }^{\circ} \mathrm{C}\right)$ and $i \mathrm{C}_{4} \mathrm{H}_{10}\left(-11.7{ }^{\circ} \mathrm{C}\right)$. However, $\mathrm{R} 134 \mathrm{a}$ and $i \mathrm{C}_{4} \mathrm{H}_{10}$ forms an azeotropic mixture, i.e. a mixture that cannot be separated taking advantage of different boiling points or by simple distillation. This happens because an azeotrope has a unique boiling point. Indeed, nothing would liquefy in the first stage (at $-25{ }^{\circ} \mathrm{C}$ ) while all the mixture condenses in the second stage (at $-35^{\circ} \mathrm{C}$ ). The R134a separation occurs in the third buffer volume kept at higher temperature (some degree Celsius above $0{ }^{\circ} \mathrm{C}$ ): the liquid R134a present at the bottom of the second stage buffer (below the azeotrope) enters the warm buffer and it evaporates again. Then, a fraction returns in the second stage, while most of the pure R134a goes out of the warm volume with a slow evaporation. After these tests, the LHC Long Shutdown 2 started and for about one year all the RPC detector systems were off for maintenance and upgrades (i.e. no mixture was available to continue the R\&D study). At the beginning of 2020, the prototype was moved to CMS and connected to the CMS-RPC detector and 
a)

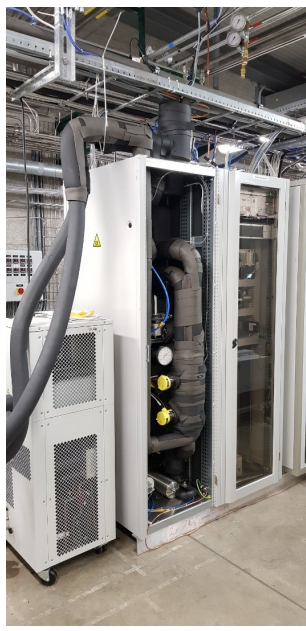

b)

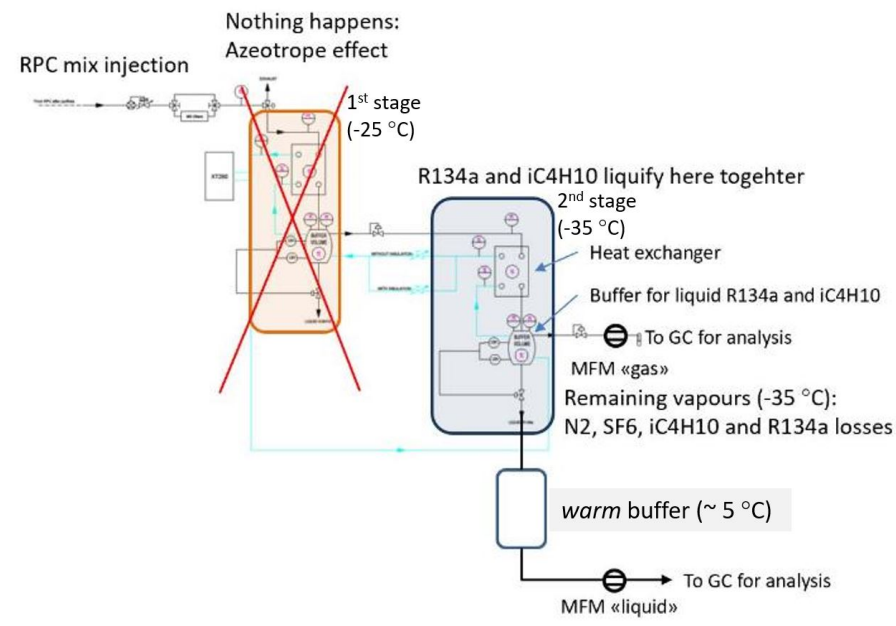

Figure 2: a) First prototype of the R134a recuperation plant tested on the ATLAS and CMS-RPC detector systems. b) Schematic view of the system with working principle.

gas systems. Several tests performed with an input gas mixture flow from 100 to 400 1/h confirmed the results obtained in ATLAS. The tests highlighted also how the equilibrium vapor-liquid present in the second stage and warm buffers between R134a- $i \mathrm{C}_{4} \mathrm{H}_{10}$ is very delicate and therefore the integration of a compressor for storage and re-use of the recuperated R134a will require further studies. Also effects related to the temperature of the warm buffer need to be better understood and, certainly, this temperature needs to be optimized and controlled. A R134a recuperation efficiency close to $85 \%$ has been achieved with a good rejection of others mixture components: only traces of $i \mathrm{C}_{4} \mathrm{H}_{10}$ and Nitrogen at ppm levels are present in the recuperated gas. Probably the current $15 \%$ loss can also be addressed and decreased. Further tests are needed to investigate the filtering capacity with respect to RPC specific impurities ${ }^{1}$. Indeed, during both test periods, these specific impurities were either barely visible (in 2018 at ATLAS, just before the end of LHC-Run2) or absent (now at CMS due to LHC-LS2) in the return RPC mixture. Given the positive results, this prototype can paved the way for the design of a final R134a recuperation system in view of the LHC Run3.

Alternative gases A third research line is based on the long-term replacement of currently used GHGs for the operation of gaseous detectors. An intense R\&D activity is ongoing in particular for the RPC detectors [8-10] which today represent the large part of the emissions at the LHC experiments. However, finding a suitable replacement for the RPC systems at the LHC experiments is particularly challenging because most of the infrastructure (i.e. high voltage systems, cables, front-end electronics) as well as the detector itself cannot be easily replaced [11-13].

GHGs disposal For cases where it is not possible to re-use/recuperate the gases, systems for the disposal of GHG have been developed by industry. They are adopted when GHG used in industrial processes are polluted to a level where recuperation for re-use is not possible. Most important,

\footnotetext{
${ }^{1}$ impurities with chemical structure very similar to the R134a present in the return gas from RPC detectors operated in presence of high radiation background
} 


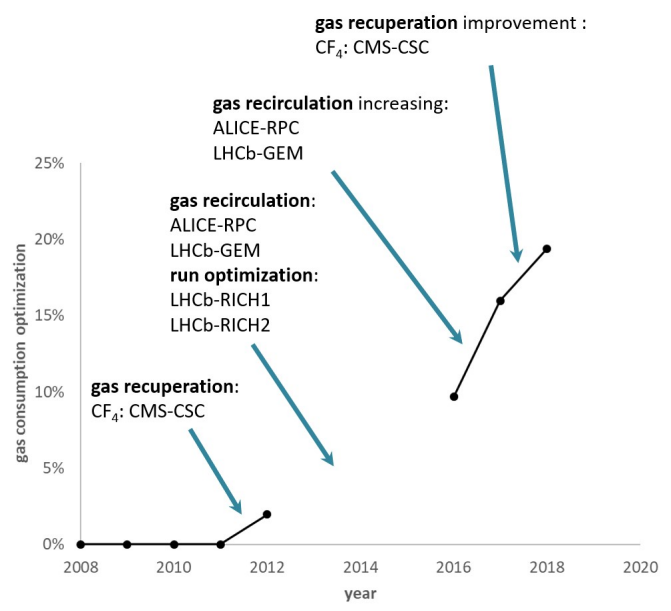

Figure 3: Optimization of GHGs consumption usage in the recent few years as a function of the upgrades performed on different LHC gas systems. In the plot, $100 \%$ optimization corresponds to a null gas consumption. Data corresponding to the period 2013-2015 are not included in the plot because not representative of normal LHC-run conditions (it was the first LHC long shutdown when most of gas systems were off for maintenance and upgrades).

GHG abatement systems are only solving the problem of the GHG emissions but they don't address issues like gas availability and price for detector operation.

\section{Conclusions}

The different strategies identified and here described should be combined to achieve maximum possible exploitation of currently used GHG. This approach allows to reduce consumption as well as operational costs and to minimize potential problems due to future availability. Gas recirculation and recuperation systems or/and the use of new eco-gases can protect against an increase of price, which is already visible in some countries. However, the challenge in using new eco-gases is coming from the fact that they behave in a slightly different way with respect to R134a and therefore current detectors and front-end electronics are not optimized for their use. Figure 3 summarizes by how much the GHGs consumption has been reduced in the recent past few years as a function of the upgrades performed on the LHC gas systems (excluding the optimization already achieved at the design phase). After LS2 a further big improvement could come from a reduction of the leak rate at detector level combined with the implementation of the R134a recuperation plant, which is now in a design and test phase.

\section{References}

[1] Regulation (EU) No 517/2014 of the European Parliament and of the Council on fluorinated greenhouse gases and repealing Regulation (EC) No 842/2006

[2] R. Guida, M. Capeans, B. Mandelli, Gas Systems for Particle Detectors at the LHC Experiments: Overview and Perspectives, Springer Proceedings in Physics (2012), 212 (2018), doi:10.1007/978-981-13-1313-4_19 
[3] B. Mandelli, R. Guida, M. Gagliardi, M. Marchisone, Gas mixture quality monitoring for the RPC detectors at the LHC experiments, XIV Workshop on Resistive Plate Chambers and Related Detectors (RPC2018), Puerto Vallarta, Jalisco State, Mexico (2018)

[4] R. Guida, M. Capeans, B. Mandelli, Strategies for reducing the environmental impact of gaseous detector operation at the CERN-LHC experiments, IEEE Nuclear Science Symposium and Medical Imaging Conference, San Diego, CA, USA.(2015), doi:10.1109/NSSMIC.2015.7581800

[5] R. Guida, M. Capeans, B. Mandelli, Strategies for reducing the environmental impact of gaseous detector operation at the CERN LHC experiments, Nucl. Instrum. Meth. A, 845 (2017) 253-256, doi:10.1016/j.nima.2016.04.067

[6] CERN Environment Report-Rapport sur l'environnement 2017-2018, Vol. 1 (2020)

[7] R. Guida, M. Capeans, F. Hahn, S. Haider, B. Mandelli, Results from the first operational period of the $\mathrm{CF}_{4}$ recuperation plant for the Cathode Strip Chambers detector at the CERN Compact Muon Solenoid experiment, IEEE Nuclear Science Symposium and Medical Imaging Conference, Anaheim, CA, USA.(2012), doi:10.1109/NSSMIC.2012.6551286

[8] A. Paoloni et al., Gas mixture studies for streamer operated Resistive Plate Chambers, RPC2016 Workshop Conference Proceeding, Journal of Instrumentation (2016), doi:10.1088/1748-0221/11/06/C06001

[9] B. Liberti et al., Further gas mixtures with low environment impact, RPC2016 Workshop Conference Proceeding, Journal of Instrumentation (2016), doi:10.1088/1748-0221/11/09/C09012

[10] R. Guida, B. Mandelli, Characterization of RPC operation with new enviromental friendly mixtures for LHC application and beyond, RPC2016 Workshop Conference Proceeding, Journal of Instrumentation (2016), doi:10.1088/1748-0221/11/07/C07016

[11] R. Guida, B. Mandelli, Development of new gas recirculation and recuperation systems for Resistive Plate Chamber operation with new environmental friendly gases, RPC2018 Workshop Conference Proceeding (2018)

[12] R. Guida, B. Mandelli, G. Rigoletti, Performance studies of RPC detectors with new environmentally friendly gas mixtures in presence of LHC-like radiation background, Nucl. Instrum. Meth. A (2019), doi:10.1016/j.nima.2019.04.027

[13] G. Rigoletti, B. Mandelli, R. Guida, Characterization of RPC detectors with LHC-like background radiation and new eco-friendly gas mixtures, RPC2020 Workshop Conference Proceeding, Journal of Instrumentation (2020), doi:10.1088/1748-0221/15/11/C11003 